\title{
Predictive value of mid-trimester cervical measurement data combined with maternal demographic characteristics for twin preterm birth at $<32$ weeks: a retrospective analysis and multicentre validation study
}

\author{
Mian Pan ${ }^{1}$, Jun Zhang ${ }^{1}$, Wenqiang Zhan², Yanling Lin ${ }^{1}$, Danlin Yang ${ }^{1}$, Li Li ${ }^{1}$, and \\ Xiaoying $\mathrm{Xue}^{1}$ \\ ${ }^{1}$ Fujian Medical University \\ ${ }^{2}$ Shanghai Jiao Tong University
}

August 7, 2020

\begin{abstract}
Objective: The purpose of this study was to develop a dynamic nomogram model to predict the risk of spontaneous preterm birth at $<32$ weeks in twin pregnancy. Design: A retrospective analysis and multicentre validation study Setting and Population: Women with twin pregnancies followed up in two tertiary medical centres from January 2017 to March 2019. Methods: Data on maternal demographic characteristics, transvaginal cervical length and funneling were extracted. The prediction model was constructed with independent variables determined by logistic regression analyses. The risk score was calculated according to the dynamic nomogram model. Main outcome measures: The risk of spontaneous preterm birth at $<32$ weeks in twin pregnancy. Results: In total, 1065 twin pregnancies were eligible for the study, of which the data of 764 cases $(92$ twin preterm cases $(<32$ weeks) and 672 control cases) were obtained from a tertiary medical centre as the training group and those of 301 cases (36 twin preterm cases ( $<32$ weeks) and 265 control cases) from the other tertiary medical centre as the external validation group. Based on logistic regression analyses, we built a dynamic nomogram model with satisfactory discrimination in both the training group(C-index: 0.856, 95\% CI: 0.813-0.899) and external validation group(C-index: 0.808, 95\% CI: 0.751-0.865). The restricted cubic splines and ROC curve supported the performance of the prediction model. Conclusions: We developed and validated a dynamic nomogram model to predict the individual probability of preterm birth in twin pregnancy at $<32$ weeks.
\end{abstract}

\section{ABSTRACT(240 words)}

Objective: The purpose of this study was to develop a dynamic nomogram model to predict the risk of spontaneous preterm birth at $<32$ weeks in twin pregnancy.

Design: A retrospective analysis and multicentre validation study

Setting and Population: Women with twin pregnancies followed up in two tertiary medical centres from January 2017 to March 2019.

Methods: Data on maternal demographic characteristics, transvaginal cervical length and funneling were extracted. The prediction model was constructed with independent variables determined by logistic regression analyses. The risk score was calculated according to the dynamic nomogram model.

Main outcome measures: The risk of spontaneous preterm birth at $<32$ weeks in twin pregnancy.

Results: In total, 1065 twin pregnancies were eligible for the study, of which the data of 764 cases (92 twin preterm cases $(<32$ weeks) and 672 control cases) were obtained from a tertiary medical centre as the 
training group and those of 301 cases (36 twin preterm cases ( $<32$ weeks) and 265 control cases) from the other tertiary medical centre as the external validation group. Based on logistic regression analyses, we built a dynamic nomogram model with satisfactory discrimination in both the training group(C-index: 0.856, $95 \%$ CI: $0.813-0.899)$ and external validation group(C-index: $0.808,95 \%$ CI: $0.751-0.865)$. The restricted cubic splines and ROC curve supported the performance of the prediction model.

Conclusions: We developed and validated a dynamic nomogram model to predict the individual probability of preterm birth in twin pregnancy at $<32$ weeks.

Funding: This research was supported by Fujian Key Laboratory of Women and Children's Critical Diseases Research [Fujian Maternity and Child Health Hospital], along with funds from Fujian Maternity and Child Health Hospital Innovation Project under Contract No.YCXZ18-21.

Keywords: Twin pregnancy, spontaneous preterm birth, dynamic nomogram, risk score, cervical length, cervical funneling

Tweetable Abstract: We developed a reliable and user-friendly prediction model for spontaneous preterm birth at $<32$ weeks in twin pregnancy. It can assists the counselling and decision-making of clinical treatment for twin pregnancy with differing risks of PTB.

\section{1| INTRODUCTION}

Complications of preterm birth (PTB) are the primary cause of death among children in the first 5 years of life, accounting for approximately $35 \%$ of deaths among new-borns and $18 \%$ of all paediatric deaths. ${ }^{1}$ Twin gestations are increasing in number and currently account for $3 \%$ of all live births and approximately $15-20 \%$ of all PTBs. ${ }^{2}$ The incidence of PTB is 6-8 times higher in twin pregnancy than in singleton pregnancy. ${ }^{3}$ Approximately $70 \%$ of twins are born preterm due to preterm premature rupture of membranes or spontaneous labour, while others result from an iatrogenic delivery indicated by monochorionicity, preeclampsia or other maternal or foetal disorders. ${ }^{4,5}$

Regarding preterm babies, longer gestational age at birth is associated with better prognosis. The EPIPAGE2 cohort study demonstrated that the survival rates at 22-26, 27-31, and 32-34 weeks of gestation are $51.7 \%$, $93.1 \%$, and $98.6 \%$, respectively. ${ }^{6}$ Compared with singletons, twins born preterm (before 32 weeks of gestation) are at twice the risk of high-grade intraventricular hemorrhage and periventricular leucomalacia. ${ }^{7}$ Therefore, better prediction, prevention, and management of PTB is necessary to improve the quality of maternal and neonate care.

To date, strategies for the prevention of PTB in twin pregnancy, such as the use of vaginal progesterone, a cervical pessary and a cervical cerclage, remain controversial or are considered to have limited effects. ${ }^{5,} 8-14$ To address the growing desire for better guidance for clinical practice, it is a prerequisite to distinguish the patients who are at an increased risk of extreme and very-PTB among the whole twin-pregnancy population, as follow-up treatment may be associated with greater benefit than workload in this high-risk population.

As a demonstrably available and reproducible method, cervical assessment with transvaginal ultrasonography has been increasingly used. ${ }^{15-17}$ Cervical shortening and cervical funneling are associated with a higher risk of preterm delivery. ${ }^{18-24}$ However, the predictive accuracy of cervical length for PTB in twin pregnancies are conflicting. Its isolated use as a screening tool has limited value due to low sensitivity. ${ }^{16,25,26}$ In addition, previous research has demonstrated that the risk of PTB is also affected by maternal demographic features, such as ethnic origin, age, primiparity, chorionicity, prepregnancy body mass index (BMI) and history of previous preterm delivery or late-term abortion. ${ }^{27-32}$ In a sense, twin gestation itself is one of the strongest risk factors for PTB. ${ }^{4,33}$ Assessment of the individual maternal prognosis requires clinicians to consider an array of maternal demographic factors and clinical variables that may be clinically challenging to synthesize. However, the relative contribution of certain characteristics to a given individual's likelihood of preterm birth and whether or how these features may interact remain poorly understood.

Thus, instead of complicating the clinicians' lives with close monitoring and administration resulting from 
an undefined or inherently subjective risk assessment, it would be useful to invest our research efforts in developing a simple and practical algorithm to calculate a risk assessment of PTB for twin pregnancies, similar to the first trimester genetic disease screening tools or the Framingham heart disease score. ${ }^{34}$

The purpose of this study is to improve our collective understanding of valuable predictive factors related to preterm birth of twin gestation. We then used these factors to develop and validate the prediction model of spontaneous PTB (SPTB) at $<32$ weeks to provide a comprehensive risk estimation as a clinical assessment tool.

\section{2 | MATERIALS AND METHODS}

\section{1| Study population}

We retrospectively collected data for 1580 consecutive women with twin pregnancies in the Fujian Maternity and Child Health Hospital (with 1000 beds and an annual delivery number of more than 20,000) and the Fujian Provincial Hospital (with 2398 beds and an annual delivery number of more than 5000) from January 2017 to December 2019; 1065 of these cases were eligible for participation.

Subjects with any of the following conditions were excluded: uncertain pregnancy date, maternal or foetal indications for iatrogenic PTB at $<32$ weeks, twin birth weight $<500 \mathrm{~g}$, gestational age at birth $<24$ weeks, genetic or structural abnormalities of either foetus, stillbirth of one or two foetuses, monoamniotic or monochorionic twin pregnancy complicated by twin transfusion syndrome (TTTS) or twin anaemia-polycythaemia sequence (TAPS), placement of cervical cerclage, incomplete maternal data, or delivery at a medical centre other than ours. Women who gave birth before 24 weeks were excluded because, in most cases, these women are likely to represent a unique subgroup of women whose cervical changes would be detected very early and extremely obvious. Additionally, these women would not have had their cervical measurement at the indicated gestational stage in our study period, which is a major part of our research. As a result, we excluded 515 patients who met the exclusion criteria, and thus, 1065 patients met the inclusion criteria (Figure 1).

We assigned 764 samples collected from the Fujian Maternity and Child Health Hospital as the training group and 301 samples collected from the Fujian Provincial Hospital as the external validation group. All samples were reassessed by two obstetricians according to the inclusion and exclusion criteria (the flowchart showing the derivation of the development cohort and validation cohort is presented in Figure 1). The institutional ethical review boards of both included hospitals approved this retrospective analysis of anonymous data, and the requirement for informed consent was waived by the ethics review boards.

\section{2| Data collection}

Data were extracted from the medical charts. Demographic characteristics included maternal age, prepregnancy body mass index (prepregnancy BMI), nulliparity, history of previous cervical surgery, history of smoking, clinical data including validation of gestational age by first trimester ultrasound, chorionicity, history of previous preterm or late abortion, complications during pregnancy, use of assisted reproductive technology, cervical length (20-24 weeks) and cervical funneling, gestational age at delivery.

Gestational age was calculated from the last menstrual period (LMP) and confirmed by the foetal crownrump length measurement at the first trimester ultrasonic scan. If a discrepancy of more than 7 days was observed, the sonographic gestational age was followed. Chorionicity was confirmed by identifying lambda and $\mathrm{T}$ signs with ultrasound imaging between $11^{+0}$ and $13^{+6}$ weeks of gestation. ${ }^{21}$

All patients underwent transvaginal cervical length (TVCL) measurements between 20-24 weeks prior to viability and at a gestational age when the optimal image of the cervix was relatively easy to capture. The TVCL measurements of all subjects were performed by experienced sonographers at our ultrasound units. The ultrasound assessment was performed to measure the length of the cervical canal from the internal os to the external os and observe whether the cervical funneling appears with patients in the lithotomy position with an empty bladder. The measurement was repeated under fundal pressure or the Valsalva manoeuvre unless severe cervical shortening was observed. ${ }^{16}$ In addition, all the ultrasonic reports were reviewed in 
detail for information on the presence, shape and length of cervical funneling; the length of the closed part of the cervix; and changes in cervical funneling and the closed part in response to increased abdominal pressure. $^{23}$

\section{3| Statistical analysis}

\section{Model development}

Quantitative data are expressed as the median (interquartile range, IQR), and qualitative data are expressed as the number (percentage). The Wilcoxon-Mann-Whitney test or Fisher's exact test was performed to measure the distribution differences of variables between the development and external validation groups. Univariate and multivariate logistic regression analysis was used to detect the correlation between clinical variables and preterm birth at 28 weeks, 32 weeks, and 34 weeks by applying a backward procedure based on the Akaike information criterion (AIC). By drawing the ROC curve of the predicted probabilities of SPTB before three gestational weeks $(28,32,34$ weeks) with multivariate meaningful variables, the prediction power for SPTB before the three gestational weeks was compared. Based on these results, a nomogram model with higher predictive performance was established.

\section{Model validation}

The performance of the nomogram models in identification and calibration was evaluated. The discriminative ability and predictive ability of the model are evaluated through Harrell's C-index, and external crowds are introduced to further evaluate the predictive value of the model. The calibration curve was analysed by drawing the predicted probability of the nomogram and the actual occurrence of SPTB. Restricted cubic splines were used to evaluate the correlation between the model's predicted score and the risk value of preterm birth. And the Kaplan-Meier curve was used to compare the differences between different risk groups. ROC curve analysis was also used to compare the prediction performance of the nomogram prediction model and the univariate model.

Statistical analyses were all performed with R 3.6.0 software (R Foundation, Vienna, Austria). A two-sided $\mathrm{P}$-value $<0.05$ was considered to indicate statistical significance.

\section{3 | RESULTS}

\section{Characteristics of the development and external validation groups}

Totally 1065 twin pregnancies were eligible for the study, of which 764 collected from the Fujian Maternity and Child Health Hospital were assigned to the training group, while 301 from the Fujian Provincial Hospital were into the external validation group (Figure 1). In the whole study population, the number of positive cases of SPTB at <28, 32, 34 and 37 weeks were 32(3.0\%), 150(13.8\%), 221(20.4\%) and 639(58.9\%), respectively.

Supplementary Table 1 lists the maternal demographic and clinical characteristics of the twin pregnancies in the training and external validation groups. There were no significant differences in these features between the training and validation groups (all $\mathrm{P}>0.05$ ), indicating that the characteristics of the training and external validation groups were similar and that subsequent external validation would be representative.

\section{Predictive factors associated with SPTB at $<32$ weeks}

In the training group, we conducted univariate and multivariate regression analysis to detect the correlations between clinical variables and probabilities of preterm delivery before 28 weeks, 32 weeks, and 34 weeks by applying the AIC-based backward procedure repectively (Table 1). Then we constructed three ROC curves for predicting SPTB according to the results of multivariate analysis. By comparing the AUCs, we found that the predicted value for SPTB at $<32$ weeks was the highest (Supplementary Figure 1). After comprehensively considering the predictive power and the number of positive cases of PTB before the there gestational weeks(the number of positive cases who delivered before 28 weeks was significantly less than 10 times that of multivariate meaningful variables), we finally chose to establish a predictive model for predicting PTB at $<32$ weeks. Multivariate logistic regression analysis ( $<32$ weeks) showed that nulliparity, 
monochorionicity, prepregnancy BMI, previous preterm birth or late abortion, cervical funneling and shorter cervical length were independent risk factors for SPTB at $<32$ weeks.

\section{Development and validation of a dynamic nomogram for SPTB at $<32$ weeks}

Based on meaningful independent factors in multivariate regression analysis, we developed a nomogram to predict the SPTB probability at $<32$ weeks (Figure 2). Each point could be determined based on the intersection of the vertical line from the variable to the point axis. Then, the total risk score was calculated by adding each variable point. The possibility of twin SPTB at $<32$ weeks could be read on the total point axis.

Furthermore, a user-friendly dynamic predicative nomogram was established and available online(https://zhanwenqiang.shinyapps.io/DynNomapp/). The dynamic nomogram conveniently provided individual probability of SPTB which was calculated automatically by the input characteristics of each subject (Supplementary Figure 2). The Harrell's concordance index value of the nomogram model in the training group was 0.856 (95\%CI: 0.813-0.899). Calibration plots demonstrated that the nomogram was well calibrated, with favourable agreement between the nomogram-predicted probabilities and the actual probabilities of SPTB at $<32$ weeks (Supplementary Figure 3A). When applied to the external validation group, the Harrell's concordance index value of the nomogram model in the external group was 0.808 (95\%CI: 0.751-0.865). The calibration curves indicated that the probability predicted by the nomogram was in good agreement with the actual observation results (Supplementary Figure 3B).

\section{Risk stratification and model performance test}

Next, based on the nomogram, the restricted cubic spline curve showed the risk escalated continuously with the increasing scores obtained from the nomogram, which supports the reliability of the model(Supplementary Figure 4). With the ROC curve, the optimal cut-off value of risk score(124.76) was calculated based on the maximum Youden index(Supplementary Figure 5). Then the cut-off value categorized the training population into the low risk group (163 twin pregnancies with risk score [?] 124.76) and the high risk groups (601 twin pregnancies with risk score $>124.76)$, respectively $(\mathrm{OR}=17.21,95 \% \mathrm{CI}(10.30-28.76), \mathrm{P}$ $<0.001$ )(Supplementary Table 2). A sensitivity of $73.91 \%$, specificity of $85.86 \%$, false positive rate of $14.14 \%$ and negative predictive value of $96.01 \%$ were reached by the nomogram model. Through the comparison of the survival curves of the high and low risk groups, we observed the probability of SPTB in the high risk group was significantly higher than that in the low risk group $(\mathrm{HR}=2.59,95 \%$ CI $(2.03-3.30), \mathrm{P}<0.001)$, and the gestational age at delivery was significantly earlier in the high risk group (Supplementary Figure 6).

Comparing the prediction performance between the nomogram model and individual predictors

In the training group, the AUC of the nomogram predicting the probability of SPTB at $<32$ weeks was 0.856 (95\%CI: 0.813-0.899), while the AUC of the six meaningful variables was 0.569 to 0.727 . The results showed that the prediction accuracy of the nomogram was significantly higher than that of any single variable(all $\mathrm{P} j 0.05)$. The external validation group also proved the advamtage of the nomogram on predictive accuracy. The AUC of the nomogram in the external verification set was 0.808 (95\% CI: 0.751-0.865), which was significantly higher than that of either single variable in the model(all $\left.\mathrm{P}_{j} 0.05\right)$ (Figure 3 ).

\section{4 | DISCUSSION}

\section{Main Findings}

In this retrospective analysis and multicentre validation study, we developed a predictive model of SPTB at $<32$ weeks based on maternal characteristics and sonographic cervical measurements to provide an accurate and comprehensive risk estimation, which can help physicians make informed treatment decisions about further administration and management of twin pregnancy as an assessment tool.

\section{Strengths and Limitations}


To our knowledge, this is the first study to develop a predictive nomogram for PTB of twin gestations and a web-based calculator to improve the approachability of the prediction model. Our model could synthesize the most relevant risk factors for PTB, such as nulliparity, pre-pregnancy BMI, history of PTB or late abortion, chorionicity, cervical funneling and cervical length, and generate a risk percentage number for each patient, which had a predictive accuracy (AUC 0.856 [95\% CI: 0.813-0.899]) significantly that was higher than that of either variable alone. The strengths of the study include the detailed, standardized data collection, high rate of follow-up and efficient statistical analysis. Based on the model, we could provide reliable risk estimation for clinical counselling, therapy decision-making, and follow-up strategies, rather than complicating the clinicians' lives with close monitoring and administration resulting from an undefined or inherently subjective risk assessment. All data pertaining to characteristics in our study can be easily obtained in the obstetric units where the cervical assessment has been well standardized, and these data were obtained by well-trained specialists throughout the study period. Moreover, external validation and restricted cubic splines supported the test performance.

On the other hand, the present study has some limitations. Most importantly, our study is limited by its retrospective design. There is a possibility of confounding bias: patients with unmeasured or unobservable factors who were excluded may represent patients at higher risk. Second, the sample number of marginal risk scores is relatively insufficient, which limits generalizability because it might not fully reflect the actual situations of patients at the highest risk and who are may be the most clinically interesting population. Last, the study population in the two centres is limited to our own population (Asian). This potential limitation may also be considered as a strength. All women included in the study were followed up and treated only in the two tertiary medical centre, which limits confounding factors associated with the heterogeneity in provider bias, such as clinicians' experience, and differences in process of monitoring and management for offering the intervention.

\section{Interpretation}

The reason why we comprehensively took all above factors into account to build the model was that the predictive performance of single cervical measurement is not satisfied. ${ }^{16,}{ }^{25,}{ }^{26}$ The mechanism of PTB in twin maternity is subjected to various mechanical stimuli (two continuously growing foetuses and the expanding uterus) and biochemical stimuli (inflammatory factors, fetoplacental signals and steroid hormones). ${ }^{35,} 36$ There seem to be structure-function associations between SPTB and cervical microstructure, geometry, and mechanical function, which are not fully understood ${ }^{37}$ Recently, researchers proposed a new conceptual framework for the theory of PTB in which a maternal clinical phenotype potentially related to a certain perinatal outcome is characterized by a series of common clinical characteristics observed during pregnancy. ${ }^{27,} 38$ Demographic factors such as age, race, BMI, history of PTB, previous uterine surgeries, smoking, and prevalence of PTB in that specific area may indicate the initial states and variations in the cervix, including geometry and mechanical function, which increase the risk of cervical insufficiency. All these risk factors have their interconnected effects and a computational framework in changing and remodelling the cervix. ${ }^{39-42}$

However, it has been argued that maternal factors or cervix geometry (including length) alone do not perform well in PTB risk assessment, primarily due to poor sensitivity. ${ }^{16,} 25,26,43$ In our study, we specifically investigated the role of cervical measurement data and maternal demographic characteristics on SPTB probability at $<32$ weeks. By doing so, we have found that nulliparity, monochorionicity, lower prepregnancy BMI, previous preterm birth or late abortion, cervical funneling and shorter cervical length confers an increased risk of PTB at $<32$ weeks and then build the nomogram.

By our predition model, clinicians can counsel the patients and define the follow-up schedule according to a patient's individual risk of PTB. Thus, the ability to identify patients at higher risk for SPTB will assist us to schedule tighter follow-ups or administer targeted interventions such as antenatal corticosteroid administration, tocolytic therapy and transfer to a tertiary medical centre and reduce overtreatment to those at lower risk. Further clinical trials are warranted to validate and improve its diagnostic performance, and the corresponding online calculator can be updated and serve as a basic screening tool for clinical practice and subsequent research. 


\section{5 | CONCLUSION}

We developed the first nomogram and online tool that accurately predicts the risk of SPTB at $<32$ weeks in twin pregnancy, whose screening performance has been found to be superior to either risk factor alone. It can provide application value to complement the existing clinical evidence, which assists the counselling and decision-making of clinical treatment for twin pregnancy with differing risks of PTB.

Conflict of interest statement: The authors report no conflict of interest. Completed disclosure of interest forms are available to view online as supporting information.

\section{Contribution to Authorship:}

MP, JZ and WZ conceived the idea of this study.

MP, JZ, WZ and YL designed the search strategy, screened abstracts and full texts.

YL, DY, LL and XX acquired the data.

JZ and WZ performed the data analysis.

All authors interpreted the data analysis and critically revised the manuscript.

All authors had the opportunity to review the manuscript, and provided their permission to publish the manuscript.

All authors agree to take responsibility for the work. The corresponding author attests that all listed authors meet authorship criteria, and that no others meeting the criteria have been omitted.

\section{Funding statement}

This research was supported by Fujian Key Laboratory of Women and Children's Critical Diseases Research [Fujian Maternity and Child Health Hospital], along with funds from Fujian Maternity and Child Health Hospital Innovation Project under Contract No.YCXZ18-21.

\section{Ethics approval}

All procedures performed in studies involving human participants were approved by the Fujian Maternity and Child Health Hospital of China Ethics Committee (Ethical approval number:2019-014), and are in accordance with the 1964 Helsinki declaration and its later amendments or comparable ethical standards. Because the dataset contained no data enabling patient identification and all women received standard care, the study was exempt from informed consent requirements.

\section{REFERENCE}

1. Oh KJ, Romero R, Park JY, Lee J, Conde-Agudelo A, Hong JS, et al. Evidence that antibiotic administration is effective in the treatment of a subset of patients with intra-amniotic infection/inflammation presenting with cervical insufficiency. Am J Obstet Gynecol. 2019; 221(2): 140 e1- e18.

2. Chauhan SP, Scardo JA, Hayes E, Abuhamad AZ, Berghella V. Twins: prevalence, problems, and preterm births. Am J Obstet Gynecol. 2010; 203(4): 305-15.

3. Martin Joyce A,Hamilton Brady E,Osterman Michelle J K et al. Births: Final Data for 2015.[J] .Natl Vital Stat Rep, 2017, 66: 1.

4. Zork N, Biggio J, Tita A, Rouse D, Gyamfi-Bannerman C. Decreasing prematurity in twin gestations: predicaments and possibilities. Obstet Gynecol. 2013; $122(2$ Pt 1): 375-9.

5. Rouse DJ, Caritis SN, Peaceman AM, Sciscione A, Thom EA, Spong CY, et al. A trial of 17 alphahydroxyprogesterone caproate to prevent prematurity in twins. N Engl J Med. 2007; 357(5): 454-61. 
6. Pierrat V, Marchand-Martin L, Arnaud C, Kaminski M, Resche-Rigon M, Lebeaux C, et al. Neurodevelopmental outcome at 2 years for preterm children born at 22 to 34 weeks' gestation in France in 2011: EPIPAGE-2 cohort study. BMJ. 2017; 358: j3448.

7. Rettwitz-Volk W, Tran TM, Veldman A. Cerebral morbidity in preterm twins. J Matern Fetal Neonatal Med. 2003; 13(4): 218-23.

8. Committee on Practice Bulletins - Obstetrics, Society for Maternal-Fetal Medicine,Practice Bulletin No. 169: Multifetal Gestations: Twin, Triplet, and Higher-Order Multifetal Pregnancies.[J] .Obstet Gynecol, 2016, 128: e131-46.

9. Schuit E, Stock S, Rode L, Rouse DJ, Lim AC, Norman JE, et al. Effectiveness of progestogens to improve perinatal outcome in twin pregnancies: an individual participant data meta-analysis. BJOG. 2015; 122(1): $27-37$.

10. Norman JE, Mackenzie F, Owen P, Mactier H, Hanretty K, Cooper S, et al. Progesterone for the prevention of preterm birth in twin pregnancy (STOPPIT): a randomised, double-blind, placebo-controlled study and meta-analysis. The Lancet. 2009; 373(9680): 2034-40.

11. Liem S, Schuit E, Hegeman M, Bais J, de Boer K, Bloemenkamp K, et al. Cervical pessaries for prevention of preterm birth in women with a multiple pregnancy (ProTWIN): a multicentre, open-label randomised controlled trial. The Lancet. 2013; 382(9901): 1341-9.

12. Merced C, Goya M, Pratcorona L, Rodo C, Llurba E, Higueras T, et al. Cervical pessary for preventing preterm birth in twin pregnancies with maternal short cervix after an episode of threatened preterm labor: randomised controlled trial. Am J Obstet Gynecol. 2019; 221(1): 55 e1- e14.

13. Li C, Shen J, Hua K. Cerclage for women with twin pregnancies: a systematic review and metaanalysis. Am J Obstet Gynecol. 2019; 220(6): 543-57 e1.

14. Jarde A, Lutsiv O, Park CK, Barrett J, Beyene J, Saito S, et al. Preterm birth prevention in twin pregnancies with progesterone, pessary, or cerclage: a systematic review and meta-analysis. BJOG. 2017; 124(8): 1163-73.

15. Berghella Vincenzo,Saccone Gabriele,Cervical assessment by ultrasound for preventing preterm delivery.[J] .Cochrane Database Syst Rev, 2019, 9: CD007235.

16. Pagani G, Stagnati V, Fichera A, Prefumo F. Cervical length at mid-gestation in screening for preterm birth in twin pregnancy. Ultrasound Obstet Gynecol. 2016; 48(1): 56-60.

17. Conde-Agudelo A, Romero R, Hassan SS, Yeo L. Transvaginal sonographic cervical length for the prediction of spontaneous preterm birth in twin pregnancies: a systematic review and metaanalysis. Am J Obstet Gynecol. 2010; 203(2): 128 e1-12.

18. Melamed N, Pittini A, Hiersch L, Yogev Y, Korzeniewski SS, Romero R, et al. Serial cervical length determination in twin pregnancies reveals 4 distinct patterns with prognostic significance for preterm birth. Am J Obstet Gynecol. 2016; 215(4): 476 e1- e11.

19. Khalil MI, Alzahrani MH, Ullah A. The use of cervical length and change in cervical length for prediction of spontaneous preterm birth in asymptomatic twin pregnancies. Eur J Obstet Gynecol Reprod Biol. 2013; 169(2): 193-6.

20. Moroz LA, Brock CO, Govindappagari S, Johnson DL, Leopold BH, Gyamfi-Bannerman C. Association between change in cervical length and spontaneous preterm birth in twin pregnancies. Am J Obstet Gynecol. 2017; 216(2): 159 e1- e7.

21. Khalil A, Rodgers M, Baschat A, Bhide A, Gratacos E, Hecher K, et al. ISUOG Practice Guidelines: role of ultrasound in twin pregnancy. Ultrasound Obstet Gynecol. 2016; 47(2): 247-63. 
22. Rust OA, Atlas RO, Kimmel S, Roberts WE, Hess LW. Does the presence of a funnel increase the risk of adverse perinatal outcome in a patient with a short cervix? Am J Obstet Gynecol. 2005; 192(4): 1060-6.

23. Mancuso MS, Szychowski JM, Owen J, Hankins G, Iams JD, Sheffield JS, et al. Cervical funneling: effect on gestational length and ultrasound-indicated cerclage in high-risk women. Am J Obstet Gynecol. 2010; 203(3): 259 e1-5.

24. Fox NS, Rebarber A, Klauser CK, Peress D, Gutierrez CV, Saltzman DH. Prediction of spontaneous preterm birth in asymptomatic twin pregnancies using the change in cervical length over time. Am J Obstet Gynecol. 2010; 202(2): 155 e1-4.

25. Melamed N, Hiersch L, Gabbay-Benziv R, Bardin R, Meizner I, Wiznitzer A, et al. Predictive value of cervical length in women with twin pregnancy presenting with threatened preterm labor. Ultrasound Obstet Gynecol. 2015; 46(1): 73-81.

26. Gyamfi C, Lerner V, Holzman I, Stone JL. Routine cervical length in twins and perinatal outcomes. Am J Perinatol. 2007; 24(1): 65-9.

27. Harville EW, Miller KS, Knoepp LR. Racial and social predictors of longitudinal cervical measures: the Cervical Ultrasound Study. J Perinatol. 2017; 37(4): 335-9.

28. Berveiller P, Rousseau A, Rousseau M, Bitumba I, Goffinet F, Rozenberg P. Risk of preterm birth in a twin pregnancy after an early-term birth in the preceding singleton pregnancy: a retrospective cohort study. BJOG. 2020; 127(5): 591-8.

29. Erez O, Mayer A, Shoham-Vardi I, Dukler D, Mazor M. Primiparity, assisted reproduction, and preterm birth in twin pregnancies: a population based study. Arch Gynecol Obstet. 2008; 277(4): 311-7.

30. Fuchs F, Senat MV. Multiple gestations and preterm birth. Semin Fetal Neonatal Med. 2016; 21(2): $113-20$.

31. Makrydimas G, Sotiriadis A. Prediction of preterm birth in twins. Best Pract Res Clin Obstet Gynaecol. 2014; 28(2): 265-72.

32. Premru-Srsen T, Verdenik I, Steblovnik L, Ban-Frangez H. Early prediction of spontaneous twin very preterm birth: a population based study 2002-2012. J Matern Fetal Neonatal Med. 2015; 28(15): 1784-9.

33. Goldenberg Robert L,Gravett Michael G,Iams Jay et al. The preterm birth syndrome: issues to consider in creating a classification system.[J] .Am. J. Obstet. Gynecol., 2012, 206: 113-8.

34. Lloyd-Jones DM, Wilson PW, Larson MG, Beiser A, Leip EP, D'Agostino RB, et al. Framingham risk score and prediction of lifetime risk for coronary heart disease. Am J Cardiol. 2004; 94(1): 20-4.

35. Shynlova O, Tsui P, Jaffer S, Lye SJ. Integration of endocrine and mechanical signals in the regulation of myometrial functions during pregnancy and labour. Eur J Obstet Gynecol Reprod Biol. 2009; 144 Suppl 1: S2-10.

36. Myers KM, Paskaleva AP, House M, Socrate S. Mechanical and biochemical properties of human cervical tissue. Acta Biomater. 2008; 4(1): 104-16.

37. Myers KM, Feltovich H, Mazza E, Vink J, Bajka M, Wapner RJ, et al. The mechanical role of the cervix in pregnancy. J Biomech. 2015; 48(9): 1511-23.

38. Villar J, Papageorghiou AT, Knight HE, Gravett MG, Iams J, Waller SA, et al. The preterm birth syndrome: a prototype phenotypic classification. Am J Obstet Gynecol. 2012; 206(2): 119-23.

39. Masho SW, Bishop DL, Munn M. Pre-pregnancy BMI and weight gain: where is the tipping point for preterm birth? BMC Pregnancy Childbirth. 2013; 13: 120.

40. Culhane JF, Goldenberg RL. Racial disparities in preterm birth. Semin Perinatol. 2011; 35(4): 234-9. 
41. van der Ven AJ, van Os MA, Kleinrouweler CE, de Groot CJ, Haak MC, Mol BW, et al. Is cervical length associated with maternal characteristics? Eur J Obstet Gynecol Reprod Biol. 2015; 188: 12-6.

42. Dijkstra K,Janssen H C,Kuczynski E et al. Cervical length in uncomplicated pregnancy: A study of sociodemographic predictors of cervical changes across gestation. $[\mathrm{J}]$.Am. J. Obstet. Gynecol., 1999, 180: $639-44$.

43. Honest Honest,Bachmann Lucas M,Ngai Cora et al. The accuracy of maternal anthropometry measurements as predictor for spontaneous preterm birth-a systematic review.[J] .Eur. J. Obstet. Gynecol. Reprod. Biol., 2005, 119: 11-20.

\section{Hosted file}

Table 1.docx available at https://authorea.com/users/349450/articles/474459-predictivevalue-of-mid-trimester-cervical-measurement-data-combined-with-maternal-demographiccharacteristics-for-twin-preterm-birth-at-32-weeks-a-retrospective-analysis-andmulticentre-validation-study

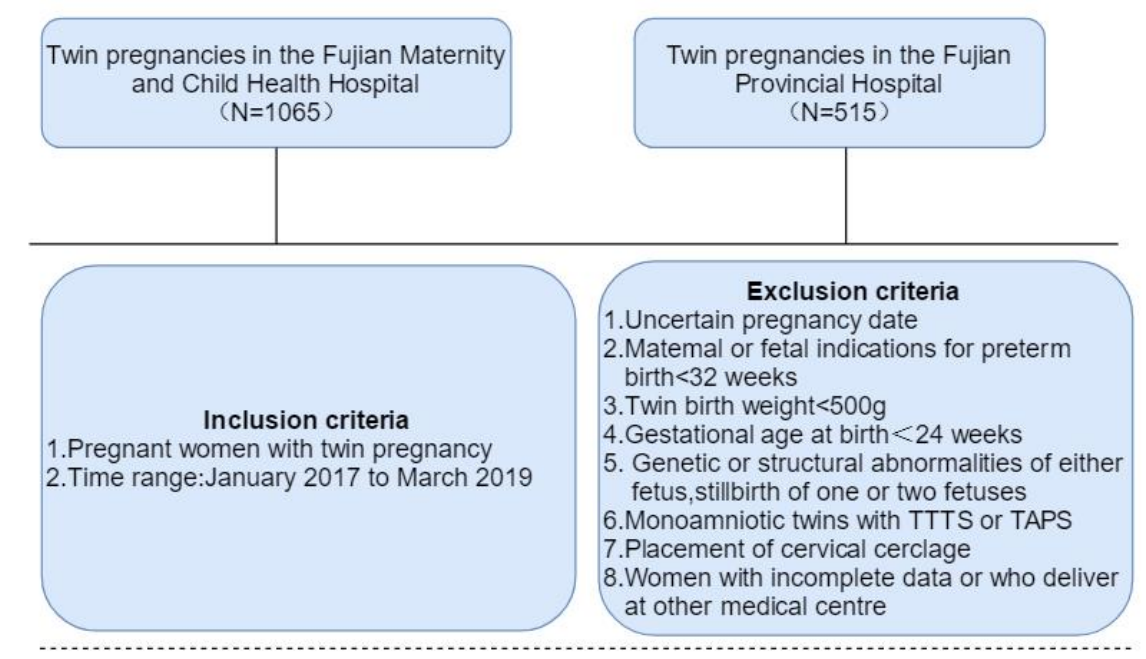

Applying Inclusion and Exclusion Critera
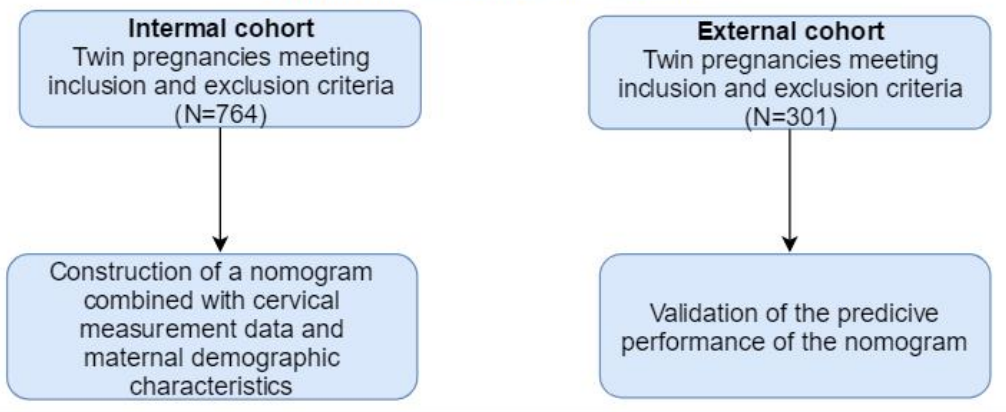

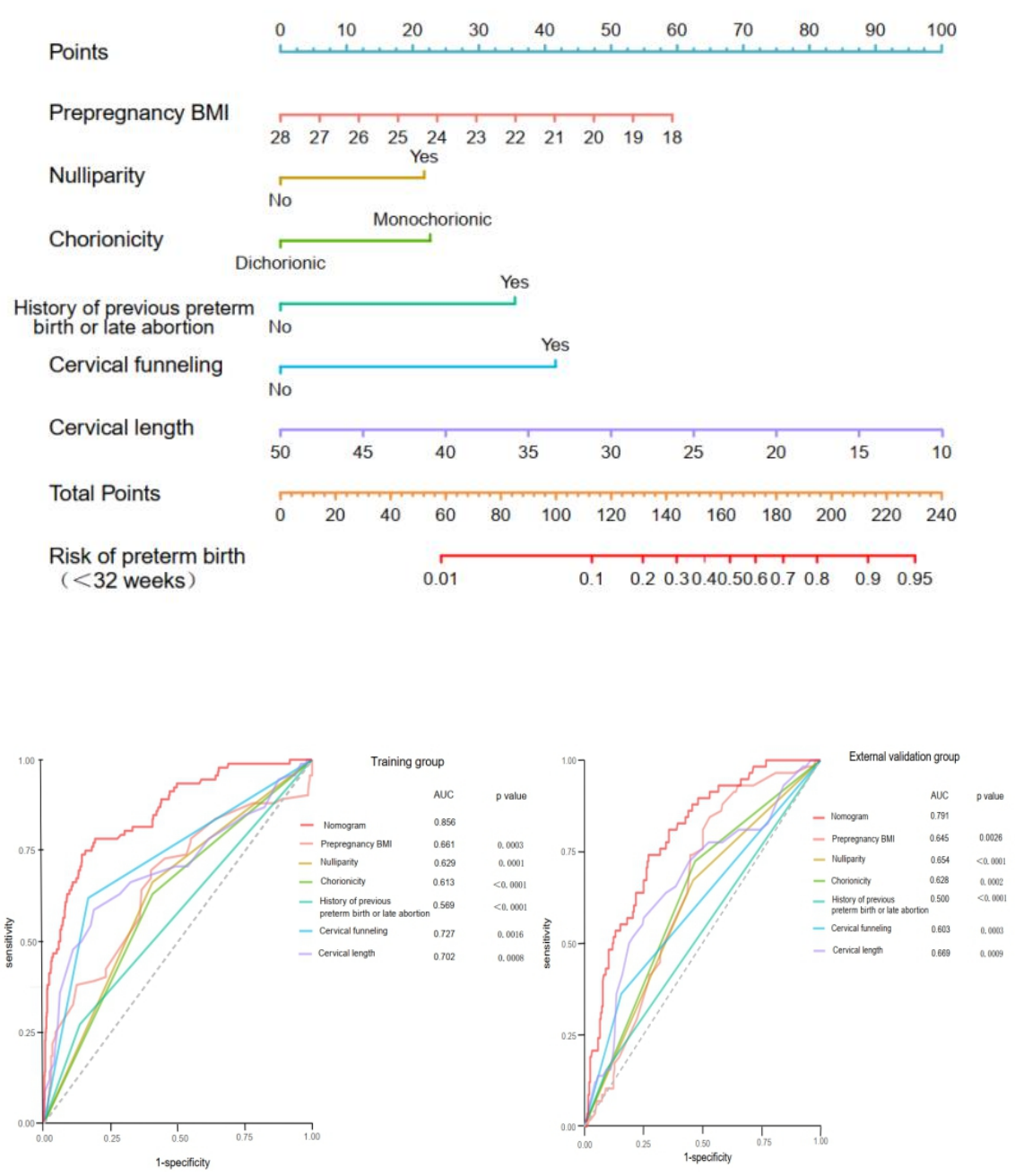